\title{
Influence of noise near blowout bifurcation
}

\author{
Peter Ashwin ${ }^{1}$ and Emily Stone ${ }^{2}$ \\ ${ }^{1}$ Department of Mathematical and Computing Sciences, University of Surrey, Guildford GU2 5XH, United Kingdom \\ ${ }^{2}$ Department of Mathematics and Statistics, Utah State University, Logan, Utah 84322-3900
}

(Received 17 March 1997)

\begin{abstract}
We consider effects of zero-mean additive noise on systems that are undergoing supercritical blowout bifurcation, i.e., where a chaotic attractor in an invariant subspace loses transverse stability to a nearby on-off intermittent attractor. We concentrate on the low noise limit and two statistical properties of the trajectories; the variance of the normal component and the mean first crossing time of the invariant subspace. Before blowout we find that the asymptotic variance scales algebraically with the noise level and exponentially with the Lyapunov exponent. After blowout it is limited to the nonzero variance of the associated on-off intermittent state. Surprisingly, for a large enough Lyapunov exponent, the effect of added noise can be to decrease rather than increase the variance. The mean crossing time becomes infinite at and after the blowout in the limit of small noise; after the blowout there is exponential dependence on the Lyapunov exponent and algebraic dependence on the noise level. The results are obtained using a drift-diffusion model of Venkataramani et al. The results are confirmed in numerical experiments on a smooth mapping. We observe that although there are qualitative similarities between bubbling (noise-driven) and on-off intermittency (dynamics-driven), these can be differentiated using the statistical properties of the variance of the normal dynamics and the mean crossing time of the invariant subspace in the limit of low noise. [S1063-651X(97)12308-X]

PACS number(s): $05.45 .+\mathrm{b}$
\end{abstract}

\section{INTRODUCTION}

On varying a parameter in a dynamical system with a chaotic attractor in an invariant subspace, the attractor can lose or gain stability at what has been termed a blowout bifurcation [1]. This bifurcation can be characterized by a Lyapunov exponent (corresponding to perturbations in a transverse direction) passing through zero. The bifurcation has been observed to occur in two scenarios; nonhysteretic, where a branch of on-off intermittent attractors limiting to the invariant subspace is created at the bifurcation [1] and hysteretic, where no nearby attractors exist near the bifurcation point.

In physical systems, such a bifurcation will not appear in any pure sense; the presence of imperfect symmetry and noise will alter the bifurcation. This has been noted by several authors; for example, Platt et al. [2] observe that the presence of noise can cause a discontinuous jump in the blowout bifurcation point. Moreover, the addition of noise to a system with an attractor in an invariant subspace before a supercritical blowout can lead to dynamics that is very different from the noise-free dynamics but qualitatively similar to the on-off intermittency that appears after the blowout; this state was called bubbling in [3]; the transition to bubbling has recently been studied in [4].

The models that have primarily been used for studies of bubbling have been random-walk-type Markov models with an elastic boundary at a fixed distance from the invariant subspace; this distance being notionally one standard deviation of the added noise from the invariant subspace [2,5-7]. In this sense the models can be thought of as dividing the phase space into two disjoint regions; one where dynamical (multiplicative) noise is dominant, away from the invariant subspace, and one where the added (additive) noise is dominant, near the subspace. The authors of $[2,6]$ obtain scalings for the escape time (i.e., from a neighborhood of the invariant subspace) and the distribution of laminar phases (length of time spent near the invariant subspace). Under the assumption of a reinjection at a constant distance from the invariant subspace they find that the escape time varies exponentially with noise before blowout (noise-induced metastability) and is essentially constant after blowout in the low noise limit.

Venkataramani et al. $[4,8]$ use a more sophisticated model, a Kolmogorov equation without an elastic barrier, to obtain scalings for fractal and spectral properties of intermittent time series in the presence of noise; they discuss both the bubbling transition and the blowout bifurcation. Note that the escape time is essentially a local property and is a measure of the random linear behavior of the system. As such it is independent of the blowout scenario.

One of the simplest statistical quantities to measure is the variance of the normal component; this has been to a large extent neglected and, as we show, the scaling properties of this can be used to characterize the system and transitions thereof in a fairly simple way for the low noise limit. The addition of noise (or the breaking of the invariance of the manifold) introduces a new type of behavior; trajectories can cross the invariant manifold. This has the effect that the two symmetry-related "stuck-on" attractors [9] are merged to form one noisy attractor (see also Lai [10] who observes attractor merging caused by the dynamics). This merging at nonzero noise is one of the most noticeable effects of the addition of noise; we derive low noise scalings for this as well.

The remainder of the paper is organized as follows: In Sec. II we consider a drift-diffusion model of Venkataramani et al. [8]. This model has three parameters corresponding to the normal Lyapunov exponent, the diffusion associated with the variation of the Lyapunov exponent and the diffusion 
associated with zero-mean additive noise. This gives a parabolic partial differential equation (PDE) for the evolution of the asymptotic probability density of deviations from the invariant subspace. The stationary density associated with this equation is obtained by solving a second-order ordering differntial equation (ODE), some asymptotic properties of this solution are investigated, in particular the scaling of the mean distance from the invariant subspace as well as the mean first crossing time of the invariant subspace. Section III presents some numerical simulations of an example mapping undergoing supercritical blowout, perturbed by additive noise; the results from here support the scalings found theoretically. In particular, although the bubbling and on-off intermittent states are qualitatively similar, they are quantitatively distinguishable by the variation of the mean first crossing time and the variance with noise level. Finally in Sec. IV we summarize and discuss our results and consider some open problems.

\section{A DRIFT-DIFFUSION MODEL}

Near blowout, in the absence of noise and over long time scales it can be shown that the dynamics in the transverse $y$ direction can be approximated by a drift-diffusion equation in a logarithmic variable $z=-\ln |y|$, see, e.g., [7]. The stationary density $P(z)$ evolves according to a forward Kolmogorov equation,

$$
\frac{\partial P}{\partial t}=\lambda \frac{\partial P}{\partial z}+\frac{\beta^{2}}{2} \frac{\partial^{2} P}{\partial z^{2}},
$$

where $\lambda$ is the normal Lyapunov exponent for the natural measure and $\beta^{2} / 2 t$ is the variance of finite-time Lyapunov exponents as $t \rightarrow \infty$. This equation can be recast in the original variables to give an equation for the probability density $Q(y)$ of the form

$$
\frac{\partial Q}{\partial t}=-\lambda \frac{\partial(Q y)}{\partial y}+\frac{\beta^{2}}{2} \frac{\partial}{\partial y}\left(y \frac{\partial(Q y)}{\partial y}\right) .
$$

Adding an extra diffusion at a rate $\sigma^{2} / 2$ to model the additive noise we get

$$
\frac{\partial Q}{\partial t}=-\lambda \frac{\partial(Q y)}{\partial y}+\frac{\beta^{2}}{2} \frac{\partial}{\partial y}\left(y \frac{\partial(\partial y)}{\partial y}\right)+\frac{\sigma^{2}}{2} \frac{\partial^{2} Q}{\partial y^{2}} .
$$

This equation was obtained by Venkataramani et al. [8]. We model the supercritical case by assuming that there are noflux boundary conditions at $y= \pm 1$.

\section{A. Stationary solutions}

If we look for stationary solutions of Eq. (2.1) we obtain the ODE

$$
\frac{1}{2}\left(\beta^{2} y^{2}+\sigma^{2}\right) \frac{d^{2} Q}{d y^{2}}+\left(\frac{3}{2} \beta^{2}-\lambda\right) y \frac{d Q}{d y}+\left(\frac{\beta^{2}}{2}-\lambda\right) Q=0 .
$$

This is solvable explicitly (see [8]) with solution

$$
Q_{s}(y)=A\left(\beta^{2} y^{2}+\sigma^{2}\right)^{(\xi-1) / 2},
$$

where $\xi=2 \lambda / \beta^{2}$. For $|y| \gg \sigma / \beta, Q_{s}(y)$ is asymptotic to $A y^{\xi-1}$ as expected from noise-free results; near the invariant subspace, $|y| \ll \sigma / \beta$, the solution is approximately $A \sigma^{\xi-1}$. The solution has branch point singularities at $y= \pm i \sigma / \beta$. We compute $A$ assuming there are no-flux boundary conditions at $y= \pm 1$ (and linear behavior up to this point) giving

$$
A(\beta, \sigma, \lambda)=\left(\int_{y=-1}^{1}\left(\beta^{2} y^{2}+\sigma^{2}\right)^{\lambda / \beta^{2}-1 / 2} d y\right)^{-1} .
$$

Thus the mean, variance, and mean absolute distance from $y=0$ are given by

$$
\begin{gathered}
E(y)=0, \\
\operatorname{var}(y)=A \int_{y=-1}^{1} y^{2}\left(\beta^{2} y^{2}+\sigma^{2}\right)^{\lambda / \beta^{2}-1 / 2} d y, \\
E(|y|)=A \int_{y=-1}^{1}|y|\left(\beta^{2} y^{2}+\sigma^{2}\right)^{\lambda / \beta^{2}-1 / 2} d y .
\end{gathered}
$$

\section{B. Scaling at blowout}

At blowout, $\lambda=0$ (so that $\xi=0$ ) and we can write $A$ $=\beta /\left[2 \sinh ^{-1}(\beta / \sigma)\right]$ to obtain

$$
\operatorname{var}(y)=\frac{\sqrt{\beta^{2}+\sigma^{2}}}{2 \beta \sinh ^{-1}(\beta / \sigma)}-\frac{\sigma^{2}}{2 \beta^{2}} .
$$

If we define $\gamma=\beta / \sigma$ then we see that

$$
\operatorname{var}(y)=\frac{\sqrt{1+\gamma^{2}}}{2 \sinh ^{-1} \gamma}-\frac{1}{2 \gamma^{2}}
$$

and so the variance depends only on the ratio $\gamma$. Moreover, for fixed $\sigma$ in the limit $\beta \rightarrow 0$ (low dynamical diffusion) we have

$$
\operatorname{var}(y)=\frac{1}{3}-\frac{2}{45}\left(\frac{\beta}{\sigma}\right)^{2}+\frac{4}{189}\left(\frac{\beta}{\sigma}\right)^{4}+O\left(\beta^{6}\right),
$$

whereas for $\sigma \rightarrow 0$ (low noise limit) we have the asymptotic behavior

$$
\operatorname{var}(y)=\frac{1}{2(\ln 2 \beta-\ln 2 \sigma)}+O\left(\sigma^{2}\right) .
$$

Note that $\operatorname{var}(y) \rightarrow 0$ as $\sigma \rightarrow 0$ but the approach has infinite derivative.

Away from the blowout, it is not so easy to get explicit expressions, but we can make progress in the low noise limit, $\sigma \ll \beta$, by approximating the stationary density (2.3) as follows:

$$
Q_{s s}(y)= \begin{cases}A \sigma^{\xi-1} & \text { for }|y|<\sigma / \beta \\ A(\beta y)^{\xi-1} & \text { for } \sigma / \beta<|y|<1\end{cases}
$$

In this case we compute

$$
A=2 \frac{\beta \lambda}{\sigma^{\xi}\left(2 \lambda-\beta^{2}\right)+\beta^{2+\xi}},
$$




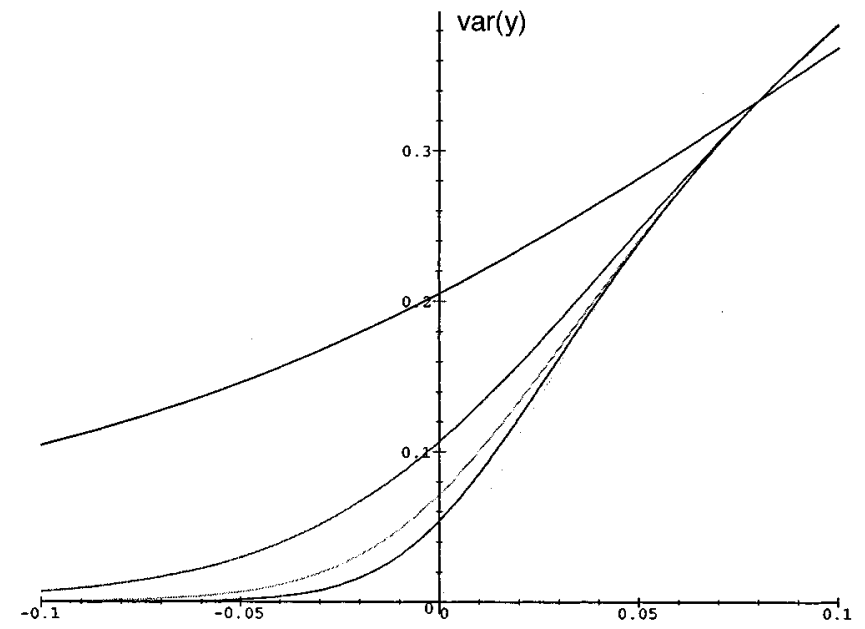

FIG. 1. Variance of $y$ plotted against $\lambda$ for $\beta=0.4$ and, from top to bottom, $\sigma=0.1,0.01,0.001,0.0001$, and 0 . This was calculated using the approximate stationary measure (2.5). Observe also that the addition of noise increases the variance up to $\lambda=\beta^{2} / 2=0.08$, after which it decreases the variance.

and hence

$$
\operatorname{var}(y)=\lambda \frac{\left(2 \lambda-\beta^{2}\right) \sigma^{\xi+2}+3 \beta^{\xi+4}}{3 \beta^{2}\left(\lambda+\beta^{2}\right)\left[\sigma^{\xi}\left(2 \lambda-\beta^{2}\right)+\beta^{\xi+2}\right]},
$$

where $\xi=2 \lambda / \beta^{2}$ as before. Note that we can compute $A$ in the limit $\lambda \rightarrow 0$ (by l'Hôpital's rule) to give

$$
A=\frac{\beta}{1+\ln \beta-\ln \sigma}
$$

\section{Scaling of the variance}

There are three distinct regions of scaling of Eq. (2.6) in the limit $\sigma \rightarrow 0$. Far from blowout, for $\lambda<-\beta^{2}$ we have

$$
\operatorname{var}(y)=\lambda \frac{1}{3 \beta^{2}\left(\lambda+\beta^{2}\right)} \sigma^{2}+O\left(\sigma^{-\xi+2}, \sigma^{-\xi}\right) .
$$

For low noise just before the blowout where $-\beta^{2}<\lambda<0$ we can compute

$$
\operatorname{var}(y)=\frac{\lambda \beta^{\xi+2}}{\left(\lambda+\beta^{2}\right)\left(2 \lambda-\beta^{2}\right)} \sigma^{-\xi}+O\left(\sigma^{-2 \xi}, \sigma^{2}\right)
$$

in the limit of $\sigma \rightarrow 0$ ( $\beta$ and $\lambda$ held constant). By contrast, for low noise after the blowout, in the region $\lambda>0$ we have the scaling

$$
\operatorname{var}(y)=\frac{\lambda}{\lambda+\beta^{2}}\left(1+\frac{\beta^{2}-2 \lambda}{\beta^{\xi+2}} \sigma^{\xi}\right)+O\left(\sigma^{\xi+2}, \sigma^{2 \xi}\right)
$$

in the limit $\sigma \rightarrow 0$ (again for fixed $\beta$ and $\lambda$ ). From this we can distinguish the blowout by the asymptotics of the variance for low noise. Schematically the variance is shown in Fig. 1
TABLE I. $\operatorname{var}(y)$ calculated using numerical approximation of the exact solution of Eq. (2.2) given by $Q_{s}$ (2.3) contrasted with the values obtained using the approximate solution given by $Q_{s s}(2.5)$. Both before and after the blowout the variances agree well to within an order of magnitude.

\begin{tabular}{ccll}
\hline \hline & & \multicolumn{2}{c}{$\operatorname{var}(y)$ computed with } \\
\cline { 3 - 4 }$\lambda$ & $\sigma$ & \multicolumn{1}{c}{$Q_{s}(y)$} & \multicolumn{1}{c}{$Q_{s s}(y)$} \\
\hline-0.05 & 0.1 & 0.1550 & 0.1458 \\
-0.05 & $10^{-5}$ & $4.383 \times 10^{-4}$ & $3.722 \times 10^{-4}$ \\
-0.05 & $10^{-10}$ & $3.284 \times 10^{-7}$ & $2.789 \times 10^{-7}$ \\
0 & 0.1 & 0.2148 & 0.2052 \\
0 & $10^{-5}$ & 0.4429 & 0.4316 \\
0 & $10^{-10}$ & 0.2193 & 0.2164 \\
0.05 & 0.1 & 0.2867 & 0.2817 \\
0.05 & $10^{-5}$ & 0.2382 & 0.2382 \\
0.05 & $10^{-10}$ & 0.2381 & 0.2381 \\
\hline \hline
\end{tabular}

computed numerically using expression (2.4); observe that it converges to the noise-free variance in a regular manner.

In particular, observe that, as predicted by Eq. (2.9), the variance is increased by low noise for $0<\lambda<\beta^{2} / 2$ whereas it is decreased by low noise for $\beta^{2} / 2<\lambda$.

For comparison of $Q_{s}$ and the approximation $Q_{s s}$, results obtained numerically using Eq. (2.4) are tabulated against those obtained using approximation (2.5) in Table I; the approximate predictions are reassuringly close although the approximations are closer after blowout than before.

\section{Mean first crossing time of $y=0$}

Adding noise to the system produces an important qualitative change in the dynamics: the appearance of trajectories that cross the invariant manifold [9]. This can be quantified by examining the mean first crossing time from $y>0$ to $y$ $<0$ for a trajectory that has reached its asymptotic statistics. Using the drift-diffusion approximation, we can estimate this by examining the flux of trajectories through zero. Note that a solution of the diffusion process that is being implicitly modeled by the Kolmogorov equation will be nondifferentiable, and crossings of $y=0$ can occur infinitely often in a finite time interval. However for randomly perturbed mappings the mean first crossing time will be well defined.

If we have $Q_{s s}(y)$, a stationary distribution and assuming that $Q_{s s}(y)$ is approximately constant for $|y|<\sigma$, we can estimate $F$, the instantaneous flux from $y>0$ to $y<0$, by

$$
F=\frac{\sigma}{2} Q_{s s}(0)
$$

(With unbiased additive noise, approximately half of all initial points in $[0, \sigma]$ will cross over within the next time unit.) If we use the approximation (2.5) for $Q_{s s}$, which predicts $Q_{s s}(y)$ is approximately constant on $|y|<\sigma$ for $\beta \ll 1$ and is valid for $\sigma \ll \beta$, we can compute

$$
F=\frac{\sigma^{\xi} \beta \lambda}{\sigma^{\xi}\left(2 \lambda-\beta^{2}\right)+\beta^{\xi+1}},
$$


and so the mean first crossing time, given by $M=1 / F$ is

$$
M=\frac{2}{\beta}+\frac{\beta}{\lambda}\left[\left(\frac{\beta}{\sigma}\right)^{\xi}-1\right]
$$

Note that this is monotonic increasing in $\lambda$, and $\xi=2 \lambda / \beta^{2}$ as before. Before blowout (i.e., for $\lambda<0$ ) this scales as

$$
M=\frac{2 \lambda-\beta^{2}}{\beta \lambda}+O\left(\sigma^{-\xi}\right)
$$

and so in particular we expect $M$ to be approximately constant. After blowout (i.e., for $\lambda>0$ ) there is an asymptotic scaling

$$
M=\frac{\beta^{1+\xi}}{\lambda} \sigma^{-\xi}+O(1)
$$

in the limit $\sigma \rightarrow 0$ for fixed $\beta$ and $\lambda$.

At blowout $(\lambda=0)$ we get the scaling

$$
M=-\frac{2 \ln \sigma}{\beta}+O(1) .
$$

Thus, at least for $\beta \ll 1$, the mean first crossing time grows algebraically in $\sigma$ and exponentially in $\lambda>0$; this growth will cause side switching to become rare for increasingly positive $\lambda$. Observe also that the mean first crossing time is unbounded at blowout as $\sigma \rightarrow 0$; this is especially interesting in that, at blowout, the variance approaches zero in the same limit.

\section{NUMERICAL EXPERIMENTS}

We now report on some numerical experiments on a smooth map undergoing a supercritical (nonhysteretic) blowout bifurcation; this map was also examined in $[2,4]$ :

$$
f\left(\begin{array}{l}
x \\
y
\end{array}\right)=\left(\begin{array}{c}
g(x)+\epsilon x y^{2} \\
\nu y e^{-\alpha x^{2}-y^{2}}+\frac{1}{2} y\left(1-e^{-y^{2}}\right)+\sigma \eta
\end{array}\right),
$$

where $(x, y) \in \mathbb{R}^{2}$ is the phase space and $\nu \in \mathbb{R}$ is a parameter. We fix on $\epsilon=0.5$ and $\alpha=0.7$ and add Gaussian noise $\eta$ with a standard deviation one scaled by a constant $\sigma$ in the $y$ variable. The map $g$ is

$$
g(x)=\frac{3 \sqrt{3}}{2} x\left(x^{2}-1\right)
$$

For various noise levels and near the point of blowout bifurcation, $\nu=1.285$, some numerically approximated stationary distributions are shown in Fig. 2.

It is interesting to observe the effect of the addition of a constant level of noise as $\nu$ passes through the blowout bifurcation at 1.285. This is shown in Fig. 3. Note that the point of blowout is quite recognizable as a point where the variance suddenly starts to grow with $\nu$; before then, the variance is governed mainly by the additive noise (recall from [11] that the attractor loses asymptotic stability at $\nu$ $=1$ ).

Figure 4 illustrates the variation of the mean first crossing time (MFCT) of $y=0$ as a function of the noise amplitude $\sigma$ and the parameter $\lambda=\nu-1.285$. The 10000 trials for any given data point were taken from a single $y$ trajectory, where each crossing of the axis was recorded after an initial integration time to remove any transients. Figure 4(a) shows the experimental MFCT vs $\ln \sigma$ before the blowout bifurcation at $\nu=1.285$, Fig. 4(b) is the variation in the MFCT vs $\lambda$ for a fixed noise level $\left(\sigma=10^{-8}\right)$, also computed numerically. In Fig. 4(c) we estimate the numerical variation of MFCT with $\sigma$ at blowout $(\lambda=0)$ with the formula computed in Sec. II. The straight line estimate shown is the $\lambda \rightarrow 0$ limit of Eq. (2.11) fitted with least squares to the data, yielding $\beta$ $=0.144$. We were also able to use the computed estimates in Sec. II to fit the MFCT variation with $\lambda$ and $\sigma$ after blowout. These are shown in Figs. 4(d) and 4(e). In Fig. 4(d) we used Eq. (2.12) with $\lambda=0.001,(\nu=1.286)$, and fitting the exponential empirically yielded $\beta=0.27$. We find that the estimates of $\beta$ fall within an order of magnitude, and the amount of variation seen is consistent with the variation of $\beta$ with the change in $\lambda$. Work is presently in progress to improve these estimates by improving the approximation (2.5).

\section{DISCUSSION}

This paper has analyzed the statistical behavior of trajectories near noise-perturbed blowout bifurcations. We have focused on two aspects; the variance of the normal coordinate and the mean first crossing time across the invariant subspace. The former is interesting in that it is a natural measure from time series, the latter is interesting in that it is only finite on the addition of noise.

In the following, $\lambda$ is the normal Lyapunov exponent, $\beta$ is the dispersion of the normal Lyapunov exponent, and $\sigma$ the noise amplitude. We find that the variance of initial conditions at the origin evolves proportional to the variance of the noise, until the asymptotic variance is approached. The variance of the normal coordinate scales to leading order as follows in the limit $\sigma \rightarrow 0$ ( $\beta$ and $\lambda$ held constant). Recall that $\xi=2 \lambda / \beta^{2}$ by definition.

(1) For $\lambda<-\beta^{2}$ we have

$$
\operatorname{var}(y) \sim \lambda \frac{1}{3 \beta^{2}\left(\lambda+\beta^{2}\right)} \sigma^{2} .
$$

(2) For $-\beta^{2}<\lambda<0$ we have

$$
\operatorname{var}(y) \sim|\lambda| \frac{\beta^{\xi+2}}{\left(\lambda+\beta^{2}\right)\left(\beta^{2}-2 \lambda\right)} \sigma^{-\xi} .
$$

(3) For $\lambda>0$ we have

$$
\operatorname{var}(y) \sim \frac{\lambda}{\lambda+\beta^{2}}\left(1+\frac{\beta^{2}-2 \lambda}{\beta^{\xi+2}} \sigma^{\xi}\right)
$$




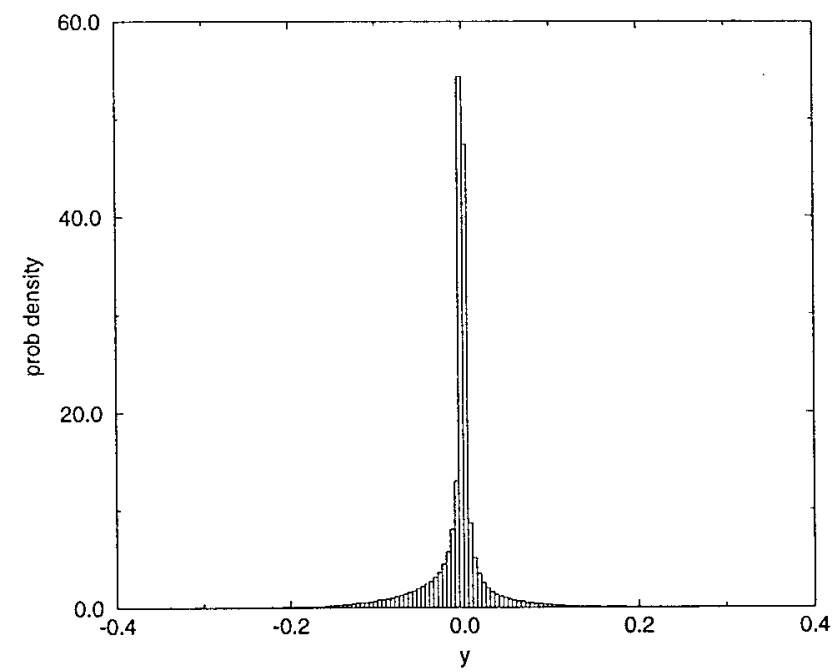

(a)

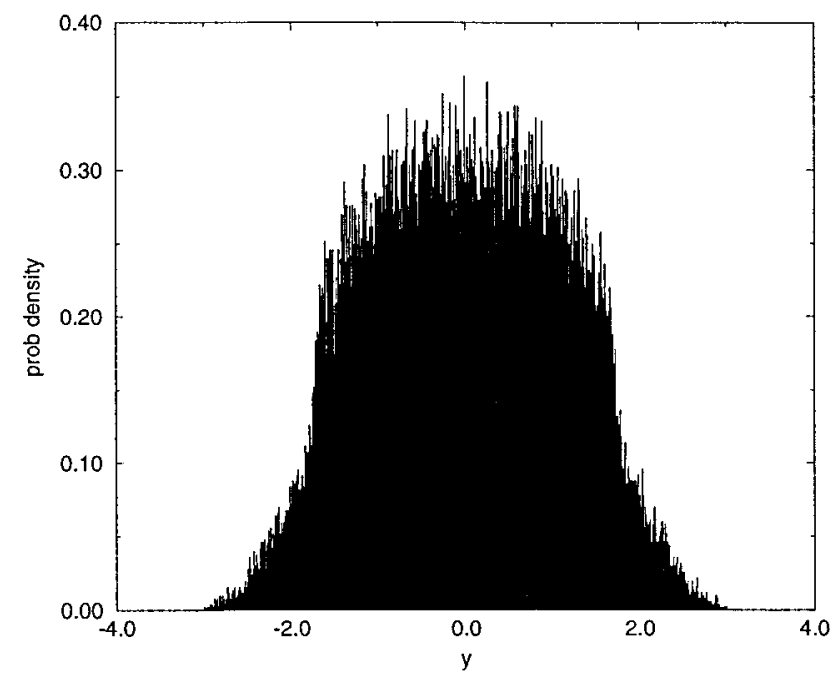

(c)

all asymptotically as $\sigma \rightarrow 0$ (again for fixed $\beta$ ). Note that the effect of low noise after the blowout is to increase variance near the blowout $\left(\beta^{2}>\lambda\right)$ but to decrease it away from the blowout $\left(\beta^{2}<\lambda\right)$.

The mean first crossing time $M$ to cross $y=0$ before blowout (i.e., for $\lambda<0$ ) scales as

$$
M \sim \frac{2 \lambda-\beta^{2}}{\beta \lambda}+O\left(\sigma^{-\xi}\right)
$$

After blowout (i.e., for $\lambda>0$ ) it scales as

$$
M \sim \frac{\beta^{1+\xi}}{\lambda} \sigma^{-\xi}+O(1)
$$

in the limit $\sigma \rightarrow 0$ for fixed $\beta \ll 1$ and $\xi=2 \lambda / \beta^{2}$. In particular the mean first crossing time diverges to infinity as the

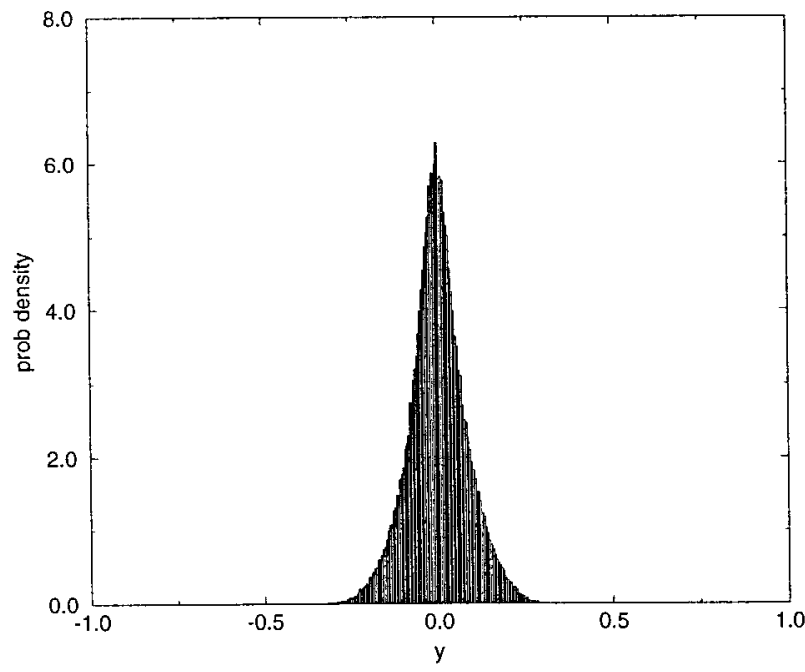

(b)

FIG. 2. Histograms of normalized stationary distributions for the normal component $y$ at $\nu=1.285$ (near the blowout bifurcation) and increasing noise level; (a) $\sigma=0.0001$, (b) $\sigma=0.01$, (c) $\sigma=1$. Note the "bell-shaped" distribution; this is predicted to have form $\left(\beta^{2} y^{2}+\sigma^{2}\right)^{-1 / 2+\lambda / \beta^{2}}$ for low noise $\sigma$ and small $y$.

amplitude of the noise tends to zero both at and after the blowout bifurcation; it is interesting to contrast this with the fact that at blowout, the variance decreases to zero with decreasing noise. All scalings in $\sigma$ have been confirmed by numerical simulations, although before blowout, the prefactors have had to be scaled.

We have only considered the effect of noise added to the $y$ or transverse component. However, Venkataramani et al. [4] note that this does not affect the scalings they find. Moreover, other results on stochastic stability [11] indicate that, at least for hyperbolic attractors in the invariant subspace, these will change in a nonsingular way on addition of low noise.

It is worth mentioning that Venkataramani et al.'s model (2.1) should be able to model several other aspects of noise perturbation near blowout, for example, the finite time development of variance of initial conditions near a subcritical blowout. Another approach using a discrete model is being developed by Nicol [13]. 


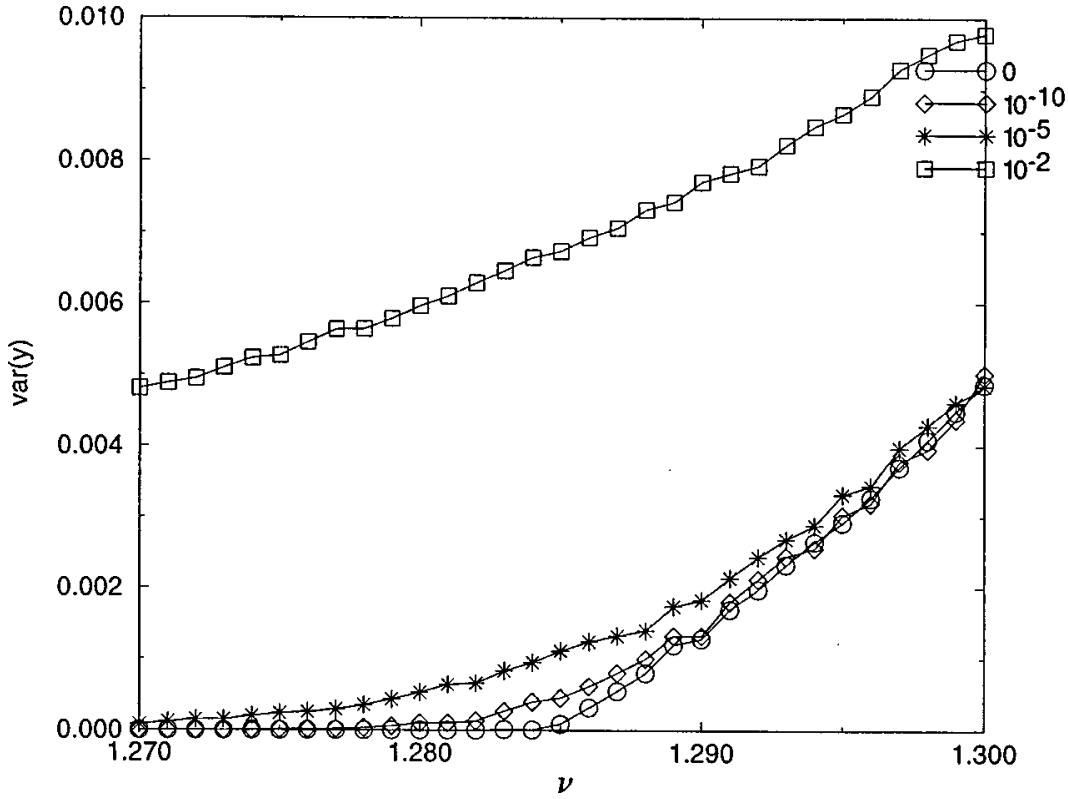

FIG. 3. Variance of the normal coordinate $y$ on varying $\nu$ through blowout bifurcation at constant noise level. 1000000 samples used for each trajectory. Observe the regular convergence to the noise-free variance which is zero before blowout and linear in $\nu$ after blowout at $\nu$ $=1.285$.

\section{Quasipotential and blowout}

The variance results in the supercritical case can be seen to extend the quasipotential for low noise perturbation to give a correction on chain recurrent sets. It can be shown that on addition of Gaussian noise of variance $\sigma^{2}$ the associated Fokker-Planck equation will have a steady distribution that scales [14] like
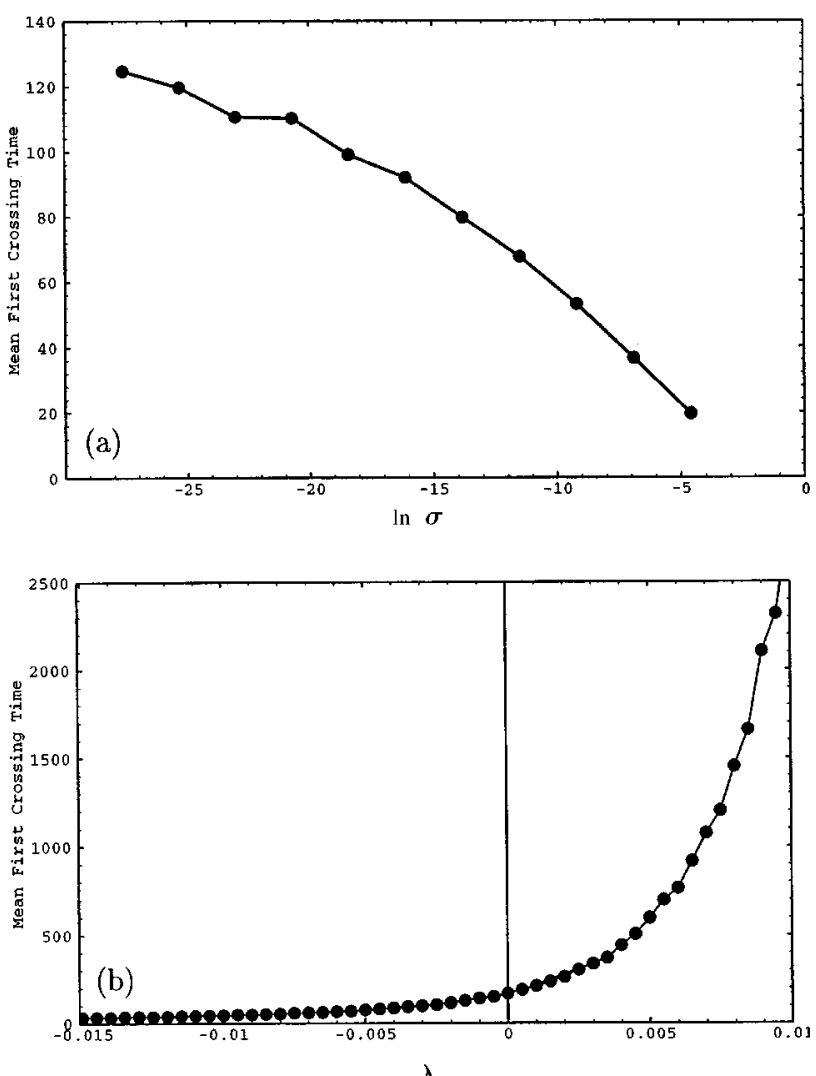

in the limit $\sigma \rightarrow 0$, where $\Phi$ is the quasipotential, a map from the phase space to $\mathrm{R}^{-}$. $\Phi(y)$ can be shown to be constant on chain-recurrent sets, and so near a blowout bifurcation where the chain recurrent set includes unstable manifolds emanating from $y=0$, we have $\Phi=0$ on a neighborhood around $y$
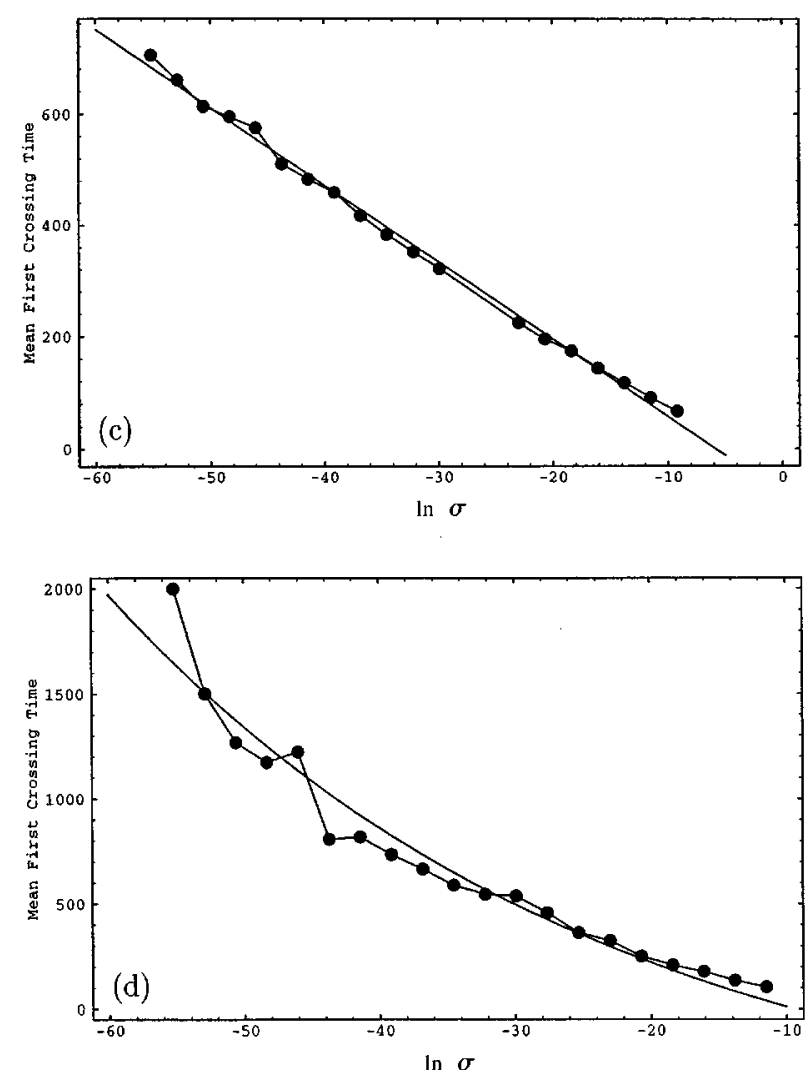

FIG. 4. Variation of the mean first crossing time $M$ of $y=0$ as a function of noise level $\sigma$ and $\lambda=\nu-1.285$, for the map (3.1). (a) $M$ vs $\ln \sigma$ before blowout, (b) $M$ vs $\lambda$ for fixed noise level $\left(\sigma=10^{-8}\right)$, (c) $M$ vs $\ln \sigma$ at $\lambda=0$, (d) $M$ vs $\ln \sigma$ after blowout. See text for explanation of the curves fitted through the data. 
$=0$. The results above indicate that in this case there is a correction to the distribution that is algebraic in $\sigma$ and takes the form

$$
y^{-1}\left(\frac{y}{\sigma}\right)^{2 \lambda / \beta^{2}}
$$

in the limit as $\sigma \rightarrow 0$. Note that this converges to zero for fixed $y>0$; however the convergence is slower than $\exp (\sigma-2)$.

\section{ACKNOWLEDGMENTS}

P.A. thanks the EPSRC for partial funding of this research. E.S. thanks the NSF for funding a visit to the UK in the autumn of 1996, out of which this work grew. We also thank Philip Aston, Paul Marriott, and especially Matt Nicol for interesting conversations with regard to this work.
[1] E. Ott and J. C. Sommerer, Phys. Lett. A 188, 39 (1994); P. Ashwin, P. J. Aston, and M. Nicol, University of Surrey Report No. 96/6/Ma, 1996 (unpublished).

[2] N. Platt, S. M. Hammel, and J. F. Heagy, Phys. Rev. Lett. 72, 3498 (1994); J. F. Heagy, N. Platt, and S. M. Hammel, Phys. Rev. E 49, 1140 (1994); N. Platt, E. A. Spiegel, and C. Tresser, Phys. Rev. Lett. 70, 279 (1993).

[3] P. Ashwin, J. Buescu, and I. N. Stewart, Phys. Lett. A 193, 126 (1994).

[4] S. C. Venkataramani, B. R. Hunt, and E. Ott, Phys. Rev. E 54, 346 (1996).

[5] A. Cenys and H. Lustfeld, J. Phys. A 29, 11 (1996).
[6] A. S. Pikovsky and P. Grassberger, J. Phys. A 24, 4587 (1991).

[7] E. Ott, J. C. Sommerer, J. C. Alexander, I. Kan, and J. A. Yorke, Physica D 76, 384 (1994).

[8] S. C. Venkataramani, T. M. Antonsen, Jr., E. Ott, and J. C. Sommerer, Physica D 96, 66 (1996).

[9] P. Ashwin, Phys. Lett. A 209, 338 (1995).

[10] Y.-C. Lai, Phys. Rev. E 53, R4267 (1996).

[11] P. Ashwin, J. Buescu, and I. N. Stewart, Nonlinearity 9, 703 (1996).

[12] L. S. Young, Ergod. Th. Dynam. Syst. 6, 311 (1986).

[13] M. Nicol (private communication).

[14] A. Hamm and R. Graham, J. Stat. Phys. 66, 689 (1992). 